\title{
Polaritonic normal-mode splitting and light localization in a one-dimensional nanoguide
}

\author{
Harald R. Haakh, ${ }^{1}$ Sanli Faez, ${ }^{1,2}$ and Vahid Sandoghdar ${ }^{1,3}$ \\ ${ }^{1}$ Max Planck Institute for the Science of Light, Günther-Scharowsky-Straße 1, D-91058 Erlangen, Germany \\ ${ }^{2}$ Debye Institute for Nanomaterials Science and Center for Extreme Matter \\ and Emergent Phenomena, Utrecht University, Princetonplein 5, 3584CC, Utrecht, The Netherlands \\ ${ }^{3}$ Friedrich Alexander University Erlangen-Nuremberg, D-91058 Erlangen, Germany
}

(Received 22 October 2015; published 21 November 2016)

\begin{abstract}
We theoretically investigate the interaction of light and a collection of emitters in a subwavelength onedimensional medium (nanoguide), where enhanced emitter-photon coupling leads to efficient multiple scattering of photons. We show that the spectrum of the transmitted light undergoes normal-mode splitting even though no external cavity resonance is employed. By considering densities much higher than those encountered in cold atom experiments, we study the influence of the near-field dipole coupling and disorder on the resulting complex super-radiant and subradiant polaritonic states. In particular, we provide evidence for the longitudinal localization of light in a one-dimensional open system and provide a polaritonic phase diagram. Our results motivate a number of experiments, where new coherent superposition states of light and matter can be realized in the solid state.
\end{abstract}

DOI: 10.1103/PhysRevA.94.053840

\section{INTRODUCTION}

The quest for understanding the interaction of light and matter has been central to the development of modern physics. Having started with ensembles of material particles and classical light more than a century ago, it is now possible to study the interaction of single photons and atoms. Here, the most prominent investigations have been performed in the strong-coupling regime of cavity quantum electrodynamics [1], where the spectrum of the atom-cavity system displays mode splitting as matter and light degrees of freedom hybridize. A well-known classical analogy to this effect is the splitting of normal modes in coupled harmonic oscillators [2].

Soon after the first reports of strong coupling, it was noted that normal-mode splitting can also be observed for a collective system, consisting of a quantum well embedded in a microcavity [3]. While this polaritonic mode splitting was observed at cryogenic temperatures, where dephasing is largely suppressed, collective strong coupling was also demonstrated in large ensembles of organic molecules coupled at room temperature despite substantial spectral broadenings [4-6]. Interestingly, similar mode splitting has even been reported for an ensemble of molecules coupled to surface plasmons and attributed to oscillator coupling [2,7,8]. In this work, we theoretically show that a propagating light field can also undergo spectral splitting when traversing a one-dimensional collection of quantum emitters, which provide feedback even in the absence of a cavity structure. Furthermore, we study the effects of disorder, density, dephasing, and mode coupling efficiency.

Recently, we pointed out that efficient coupling of a propagating photon to a single atom could be achieved by matching the latter's dipolar radiation mode, e.g., via tight focusing $[9,10]$. An alternative approach is to modify the atomic emission pattern by using planar antennas $[11,12]$ or subwavelength waveguides (nanoguide) as cylindrical antennas [13-15]. The nanoguide configuration has the decisive advantage in that it is possible to realize simultaneous coupling to a large number of emitters since it maintains the strongly confined mode over its entire length. The high versatility of the concept has been proven in several experiments that coupled light to single quantum dots [16-18], atoms [19-21], color centers [22], molecules [15,23], and superconducting qubits [24,25].

In the following, we show that this simple system is very rich in physics and focus on two key findings: First, multiple scattering can result in cavity-free polaritonic mode splitting in ensembles [2,7] (Sec. III). Second, it leads to the persistence of state localization [26-31] despite photon loss to the three-dimensional environment (Sec. IV). The new phenomena discussed in our work appear at high emitter densities, which are not easily accessible to cold atoms but feasible for emitters immersed in solid-state environments. We provide a polaritonic phase diagram to elaborate on the richness of the system and to make a link to other related works in the literature on one-dimensional ensembles.

\section{FORMALISM}

Our theoretical formalism is based on the investigation of light propagation through a large number $(N)$ of pointlike two-level quantum emitters placed in a dielectric nanoguide (see Fig. 1). To facilitate the discussion, we assign one emitter to each site $n$ of a regular lattice with spacing $L$ along the waveguide axis. We account for a general disordered system by introducing a displacement $\left|l_{n}\right| \leqslant l$ for each site. All emitters have the same resonance frequency and radial dipole moment orientation and are characterized by the polarizability

$$
\alpha(\Delta) \approx \frac{1}{\Delta-i \Gamma / 2},
$$

where $\Delta=\omega_{\mathrm{A}}-\omega_{\mathrm{L}}$ represents the detuning between the frequencies of the emitter's resonance $\left(\omega_{\mathrm{A}}\right)$ and the driving field $\left(\omega_{\mathrm{L}}\right)$. The natural linewidth $\Gamma=2 \operatorname{Im}[G(0)]$ includes the radiation reaction [32] in the nanoguide through the exact dyadic Green's function $G(z)$ [33] (see Appendix A for details). Note that despite the linear arrangement of emitters, the system is intrinsically three dimensional and photons can be lost. The emitter-nanoguide coupling is characterized by the parameter $\beta=\Gamma_{\mathrm{ng}} /\left(\Gamma_{\mathrm{ng}}+\Gamma_{\text {out }}\right)$, where $\Gamma_{\mathrm{ng}}$ and $\Gamma_{\text {out }}$ denote 


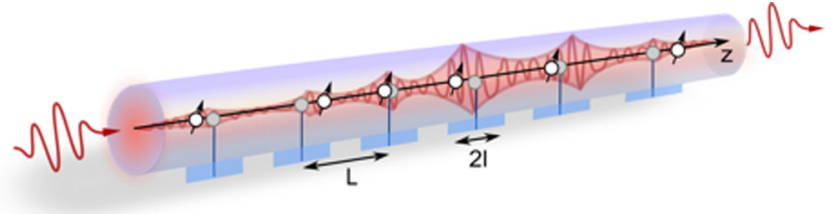

FIG. 1. Propagation of light through an ensemble of quantum emitters inside a nanoguide. The emitters can be displaced within a region $\pm l$ (blue shading) around a regular lattice of spacing $L$ (gray dots).

the single-emitter emission rates into the nanoguide mode and the outside world, respectively.

In the waveguide, a single dipole $d_{n}$ generates a field $E_{n}^{\operatorname{dip}}(z)=G\left(z-z_{n}\right) d_{n}$ [33] so that the dipole moment at each site induced by an external field $E^{\text {ext }}(z)$ and by other sites is given by $[26,27]$

$$
d_{n}=\alpha_{n}\left[E^{\mathrm{ext}}\left(z_{n}\right)+\sum_{m \neq n} E_{m}^{\mathrm{dip}}\left(z_{n}\right)\right]=\sum_{m} \mathcal{A}_{m n} E_{m}^{\mathrm{ext}} .
$$

Equation (2) fully describes the multiply scattering system. $\mathcal{A}$ acts as a collective polarizability and is obtained by the explicit inversion of the non-Hermitian matrix

$$
\mathcal{A}_{m n}^{-1}=\delta_{m n} / \alpha_{n}-G\left(z_{n}-z_{m}\right)\left(1-\delta_{m n}\right) .
$$

The real and imaginary parts of the complex eigenvalues $(\delta-i \gamma / 2)$ give the shift of the collective resonance from that of the single emitter and its linewidth, respectively. The $N$ component mode functions $\Psi=\left(d_{1}, d_{2}, \ldots, d_{N}\right) / \sqrt{\sum_{n}\left|d_{n}\right|^{2}}$ describe normalized self-sustained dipole moment distributions along the chain. For each collective (polariton) mode function, the participation number $p=\sum_{n=1}^{N}\left|\Psi_{n}\right|^{4}$ counts the number of emitters $1 \leqslant p \leqslant N$ that contribute significantly, quantifying the extension of the state [29].

\section{CAVITY-FREE POLARITONIC SPLITTING}

We now show that for large enough densities, the collective coherent interaction of the emitters with the single guided mode of the waveguide leads to the hybridization between the ensemble and the nanoguide mode.

To start our case studies, let us consider only two emitters placed in a waveguide at a separation much larger than $\lambda$. These form the mirrors of an optical resonator, where reflection is replaced by the scattering from an emitter into the waveguide mode, which, due to the good mode matching, can be highly efficient [13-15]. Unlike in a usual Fabry-Pérot cavity, however, these mirrors interact with each other via the single mode of the one-dimensional nanoguide if they are close enough compared to the photon coherence length of about $c / \Gamma[34,35]$. Here, the delocalized collective states give rise to the mode functions $\Psi_{1,2}=(1, \pm 1) / \sqrt{2}$, the collective resonances are shifted with respect to the original resonances by $\delta_{1,2}=\Delta \pm \operatorname{Re}\left[G\left(z_{1}-z_{2}\right)\right] \propto \beta \Gamma$, and their linewidths are modified to $\gamma_{1,2}=\Gamma \pm \operatorname{Im}\left[G\left(z_{1}-z_{2}\right)\right]$ due to subradiant and super-radiant decay. If near-field coupling is absent, splitting
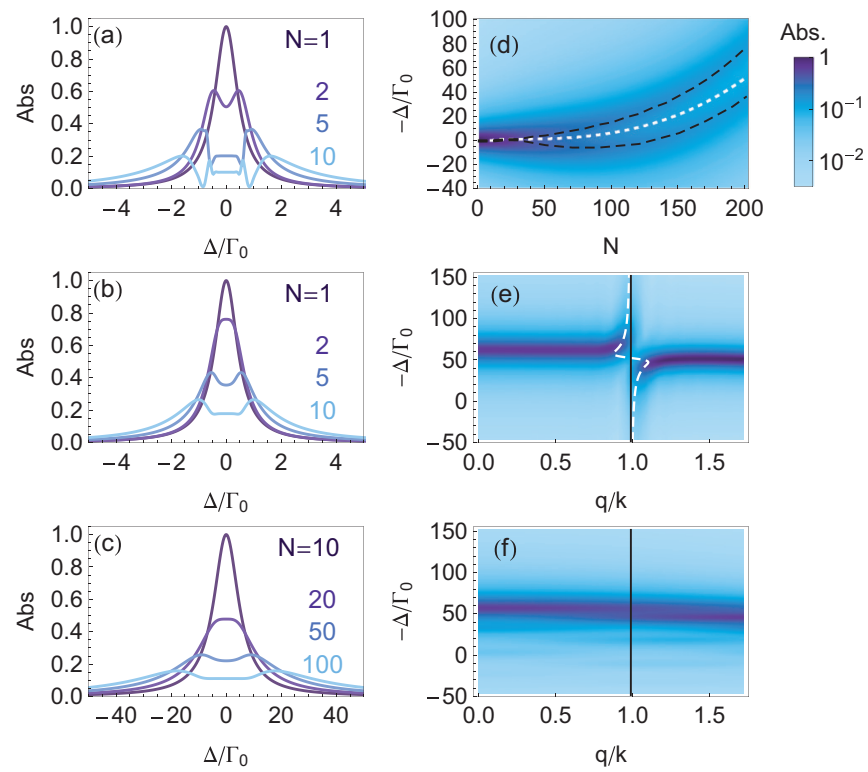

FIG. 2. (a) Absorption spectrum of an ordered chain of $N=$ $1,2,5,10$ emitters (dark to light hues) placed at separations $L=$ $2.75 \lambda$ in a nanoguide with $\beta=0.99$. The signal is normalized to the absorption signal by $N$ uncoupled resonant emitters. For $N=10$, narrow peaks near $\Delta \approx 0$ arise from subradiant modes. (b) Same as in (a) but for $\beta=0.56$. (c) Same as in (a) but for chains of $N=10,20,50,100$ emitters and with an additional homogeneous broadening of $\Gamma_{\text {deph }}=10 \Gamma_{0}$. (d) Density-dependent splitting: absorbed power as a function of the frequency detuning of the driving field and the number $N$ of emitters. The nanoguide ( $\beta=0.56, l=0)$ is now filled to a constant length of $N L=7.7 \lambda$ by a varying number of emitters. Homogeneous broadening is taken to be $10 \Gamma_{0}$. The white dotted curve plots the near-field shift, while the black dashed curves trace the absorption maxima. (e), (f) Dispersion relations obtained by plotting the absorbed power as a function of the wavenumber $q$ of the driving field and its detuning for a dense ordered chain with $L=0.04 \lambda$ and $\Gamma_{\text {deph }}=10 \Gamma_{0}$ for $N=200$ (e) and $N=10$ emitters (f). In the former case hybridized excitations approach the dispersion relation of the nanoguide (solid black curve) and agree with a mean-field model (dashed white curve, cf. Appendix B).

is thus limited to one linewidth, cf. Fig. 2(a) (cf. Appendix C for details).

If we now insert $N$ emitters, multiple scattering and the resulting collective modes yield a larger frequency splitting, which increases with $N$. Figure 2(a) illustrates the frequencydependent absorption of the system $\left(\propto \sum_{m} \operatorname{Im}\left[d_{m} E^{\text {ext* }}\left(z_{m}\right)\right]\right)$ for $N=1,2,5,10$ emitters spaced at $L=2.75 \lambda$ in a nanoguide with $\beta=0.99$. These curves clearly show that one can achieve significant normal-mode splitting from an ensemble of emitters even if they are not coupled to any external resonant structure such as a cavity. As it turns out, multiple scattering between the emitters actually generates several resonances, whose interferences create additional weaker Fano-like resonances.

We identify the fundamental underlying mechanism in the observed phenomenon to be the efficient coupling of all emitters to a single spatial mode and, thus, their coherent interaction with each other. To this end, one can also recognize the main role of a microcavity [3,7] or surface plasmon [2,36,37] to 
be to offer a mode with high $\beta=F /(F+1)$ for efficient multiple scattering from many emitters, where $F$ is the Purcell factor [38]. In this point of view one would expect a clear dependence on the degree of coherence of the emitters' transition and on the coupling efficiency $\beta$, i.e., the effect of spatial mode matching and the leakage out of the nanoguide mode. In Fig. 2(b), we plot the calculated absorption as in Fig. 2(a) but for a reduced value of $\beta=0.56$. It is clear that the normal-mode splitting is substantially weakened. Similarly, the smaller resonances are reduced to a background.

Another source of coherence loss is dephasing in the condensed phase, which is particularly significant at room temperature. As a basic model for dephasing, we allow for additional homogeneous broadening beyond the natural linewidth $\Gamma_{0}=2 \operatorname{Im}[G(0)]$, by introducing an additional rate $\Gamma_{\text {deph }}=10 \Gamma_{0}$ in the polarizability. As displayed in Fig. 2(c), the splitting is indeed washed out for $N=10$. However, we see that the loss of coherence can be compensated by increasing the number of emitters. Here, it should be borne in mind that due to the finite coherence length $c / \Gamma$ of the emitted photons, not only the total number $N$ but also the density is an important measure. Figure 2(d) shows that large frequency splittings occur in the absorption spectrum of a nanoguide when the density is increased over a fixed total length of 7.7 $\lambda$. The white dotted curve in this figure points out a general shift $\approx-\operatorname{Re}[G(L)]$ that accompanies the mode splitting and is caused by near-field interactions among neighboring emitters.

A decisive indicator of the collective hybridization with the nanoguide mode is a repulsive splitting in the dispersion relation [2]. Figure 2(e) shows the absorption of a nanoguide containing $N=200$ emitters driven by a field $E^{\text {ext }}(z)=E_{0} e^{i q z}$ as a function of the frequency detuning $\Delta$ and wave number $q$. Experimentally this is feasible, e.g., in a Kretschmann configuration. Although we have assumed a sizable dephasing of $\Gamma_{\text {deph }}=10 \Gamma_{0}$, one clearly observes a splitting of the absorption maximum into two branches along the dispersion curve $k(\Delta)$ of the empty waveguide. We note in passing that the frequency dependence of the latter is negligible since $\omega_{A} / \Gamma_{0} \approx 10^{6}$. To illustrate the importance of the density, in Fig. 2(f), we show the same calculation for $N=10$. In this case, there is no clear avoided crossing, but several weak maxima arise at various $q$ values.

In our approach, splitting arises from a microscopic description of scattering in the medium. When cavity-free mode splitting was reported for an ensemble of molecules coupled to surface plasmons [8], the collection of emitters was modeled with an effective polarizability [2]. The two approaches can be reconciled when multiple scattering is not prevalent and can be described as a mean-field effect (Appendix B). This and all results of this section remain qualitatively valid in moderately disordered samples as the relatively high value of $\Gamma_{\text {deph }}$ reduces the impact of multiple coherent scattering.

\section{COLLECTIVE STATES, MULTIPLE SCATTERING, AND A PHASE DIAGRAM}

We now elaborate on the rich character of the polaritonic states, their effects on the propagation of light, the role of disorder [39], and near-field couplings. These result in Bragg reflection, longitudinal state localization, and its eventual collapse to two-body resonances. These effects are summarized in a polaritonic phase diagram.

To set the ground, we first consider ordered chains $(l=0)$ with large spacings $L>\lambda$, where near-field effects are negligible and $\Gamma_{\text {deph }} \ll \Gamma$. Here, interactions are mediated by the guided mode with wave number $k=2 \pi / \lambda$ and $G(z) \approx$ $i \beta \operatorname{Im}[G(0)] e^{i k|z|}[40,41]$ and multiple coherent scattering affects the system properties. For even $N$ and Bragg or anti-Bragg conditions (odd or even $2 L / \lambda \in \mathbb{N}$, respectively), $\mathcal{A}^{-1}$ can be exactly diagonalized by a Fourier ansatz (cf. Appendix D). One of the resulting eigenstates is super-radiant with the linewidth

$$
\gamma_{+}=[1+\beta(N-1)] \Gamma
$$

and a mode function $\Psi=(1,1, \ldots) / \sqrt{N}$ (even $2 L / \lambda$ ) or $\Psi=(1,-1, \ldots) / \sqrt{N}$ (odd $2 L / \lambda)$ that is perfectly matched to the waveguide mode. Scattering from this state alone yields efficient reflection and ohmic transmission $T \propto N^{-2}$ as $N \rightarrow \infty$, in agreement with previous results [42], and can be understood as coherent extinction by a collective superdipole. The remaining $N-1$ eigenstates are degenerate and subradiant with

$$
\gamma_{-}=(1-\beta) \Gamma
$$

Their mode functions are periodic but do not match the guided mode and cannot be excited through the nanoguide. Since the $N$ states provide a complete basis, we make the important observation that $\gamma_{-}$and $\gamma_{+}$describe general bounds for the decay rates in far-field coupled chains independent of the exact arrangement.

At different periodicities, extended Bloch-like states arise. Figure 3(a) shows the squared amplitude of exemplary extended mode function $(p=142)$ in a lattice of $N=250$ emitters with $L=2.75 \lambda$ and low $\beta$ value of 0.4 . In Fig. 3(b), we identify the complex eigenvalues of the emitters on the real (frequency shift) and imaginary (linewidth) axes, revealing a band gap around the single-emitter resonance $(\delta=0)$. We see that subradiant extended modes with decay rates $\gamma_{-} \leqslant \gamma<\Gamma$ gather at the band edges and that all collective modes involve a large number of emitters $(p \approx 100, \ldots, 200)$ as indicated by the color-coded participation number assigned to each resonance.

The more general case of a dielectric medium involves an intrinsic degree of disorder [29,43]. Therefore, we now set $l=\lambda / 2$ to provide full phase randomization while considering a chain of lattice spacing $L=2.75 \lambda$ and $\beta=0.4$ as before. In Fig. 3(c) we present two examples of the resulting mode functions. The light (blue) curve displays a super-radiant mode that preserves the extended nature of its ordered counterpart in Fig. 3(a), while the dark (purple) curve shows the characteristic exponential envelope of a localized state. In Fig. 3(d) we plot the complex eigenvalues of the various modes and show that the localized states with $p \ll N$ gather in the subradiant sector, while the decay rates remain confined between $\gamma_{ \pm}$. We note that subradiant states move into and eventually close the band gap as $l$ increases, but-being subradiant, i.e., dark-they do not significantly modify the optical response in the band gap region. In fact, the poor spatial match of exponentially confined 

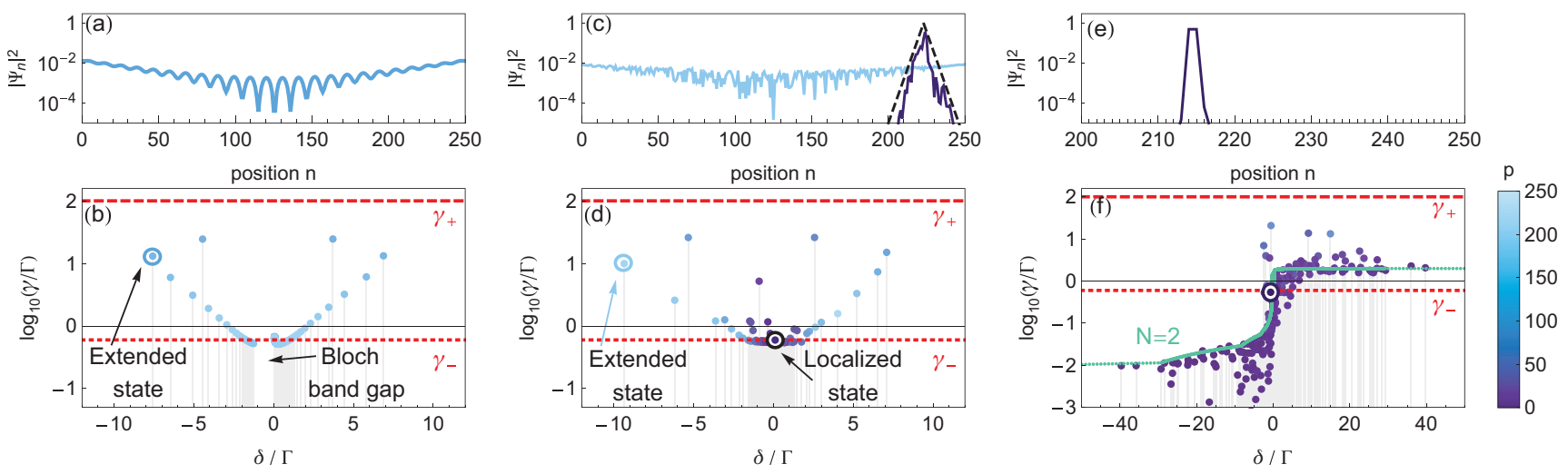

FIG. 3. Chains of $N=250$ emitters coupled to a nanoguide at low $\beta=0.4$. (a), (b) Ordered chain with $L=2.75 \lambda$, $l=0$. (c), (d) Disordered dilute chain with $L=2.75 \lambda, l=\lambda / 2$. (e), (f) Disordered dense chain with $L=\lambda / 2 \pi, l=0.49 L$. The color coding gives the participation number of the respective mode function. (a) Exemplary mode function of an extended state. (b) The 250 eigenvalues forming the polariton spectrum plotted as a function of their real $(\delta$; horizontal) and imaginary ( $\gamma$; vertical) parts. The marked extended state corresponds to the mode function in (a). (c) Exemplary mode functions of an extended state (light blue) and a localized state (dark purple). The black dashed curve shows an exponential envelope for comparison. The corresponding states are marked by the circles in (d). (d) gives the polariton eigenvalues with subradiant localized states $(\gamma<\Gamma, p \ll N)$ filling the band gap. (e) Exemplary mode function of a two-body resonance, corresponding to the state marked in (f). (f) Polariton eigenvalue spectrum. The green curve displays the eigenvalues of a pair states ( $p=2$ ) on a minimal chain of $N=2$ emitters placed at different distances.

modes to the nanoguide mode provides only little scattering, mainly out of the guide.

The combination of subradiance, reduced participation number, and exponential mode envelopes reported above clearly indicate that longitudinally localized polaritons persist for open systems with $\beta<1$. This finding was, to our knowledge, not communicated previously. Furthermore, the existence of a lower bound $\gamma_{-}$to the linewidth according to Eq. (5) [cf. Fig. 3(d)] suggests that transverse loss to the three-dimensional (3D) continuum limits the polariton lifetime regardless of the number of scatterers. As a result, the system is not in the regime of Anderson localization in the strict sense because the Thouless criterion [44], which predicts ever stronger spectral line narrowing as the particle number $N$ increases, remains indecisive unless $\beta \rightarrow 1$. This situation differs fundamentally from the 3D arrangements of emitters where a phase transition may occur [45,46] and also from lossless true 1D systems, which always show Anderson localization [44].

We have seen that losses can be compensated by increasing the number of emitters within the coherence length $c / \Gamma$. However, at very high densities $(L \ll \lambda)$ and/or strong disorder $(L-l \ll \lambda)$, neighboring emitters are coupled by a nearfield potential $G(z) \propto-1 / z^{3}$ [46-48]. Here, next-neighbor interactions dominate over the nanoguide-mediated coupling to other emitters, leading to the fractionalization of the medium into independent chainlets of neighboring pairs even at moderate disorder, with properties resembling the initial example in Sec. III (see also Appendix C). Figure 3(e) displays the pair character of the eigenstates in an exemplary mode function for a chain of $N=250, L=\lambda / 2 \pi$, and $l=0.49 L$. In this regime, the general limit $\gamma_{-} \leqslant \gamma \leqslant \gamma_{+}$no longer applies, and the polariton spectrum shows a significant number of strongly subradiant states with decay rates that are several orders of magnitude below $\gamma_{-}$[see Fig. 3(f)]. These sharp resonances generate distinct features in the optical response.
The dominant (purple) hues in Fig. 3(f) show that nearly all polariton modes are strongly confined to $p<20$ emitters. In fact, the participation number distribution strongly peaks at $p=2$, and the states gather near the green curve obtained for a chain of $N=2$ emitters placed at different spatial separations. While $G(z)$ can acquire any complex phase in the guided far field, $\operatorname{Im}[G(z)] \approx \Gamma_{0} / 2$ in the near field so that for each pair of emitters the sub- and super-radiant states with frequency shifts $\delta_{1,2}$ and line widths $\gamma_{1,2}$ are red- and blue-detuned, respectively $[46,47]$. This leads to the strongly asymmetric shape of the spectrum in the complex plane.

We present a polaritonic phase diagram in Fig. 4: as the lattice spacing $L$ and disorder amplitude $l$ are varied, the

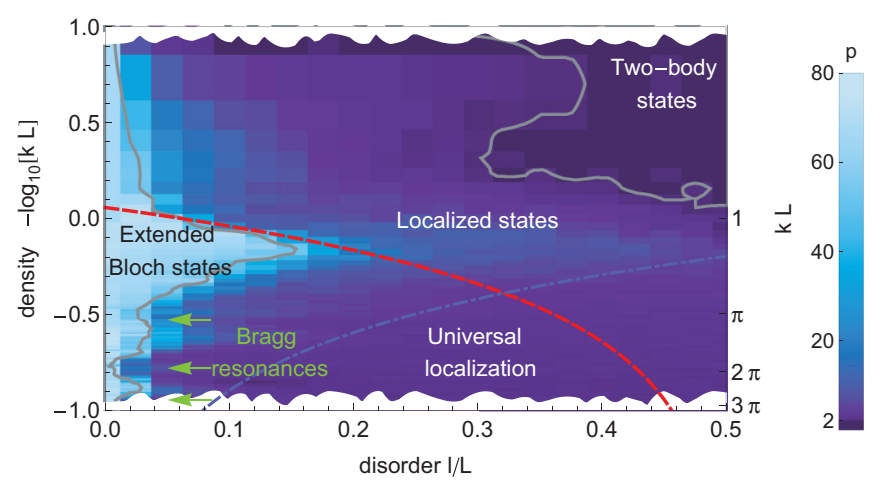

FIG. 4. Polariton phase diagram obtained from the average participation number $p$ as a function of lattice constant $L$ and disorder amplitude $l$ for a chain of $N=100$ emitters in a nanoguide $(\beta=0.4$, averaged over 10 realizations). Near field coupling can affect next neighbors above the (red) dashed curve. Below the (blue) dash-dotted curve, phase randomization leads to a universal behavior. The (green) arrows indicate the first Bragg resonances. To indicate the regions ruled by extended states and two-body resonances, respectively, the contours $p=50$ and $p=2.5$ provide guides to the eye. 
color coding gives the participation number $p$ per eigenstate of a chain of $N=100$ emitters, averaged over all eigenstates and several realizations. For ordered systems, the light (blue) hues indicate extended states. Among these, Bragg resonances (green arrows) appear as local dips because only the bright state is optically active. Extended states are rapidly suppressed by disorder at the onset of near-field coupling between next neighbors $(|L-l|<\lambda)$ above the dashed (red) curve. As the disorder amplitude increases, a crossover leads to longitudinally localized states (purple regions). Full phase randomization is reached below the dot-dashed (blue) curve. The intersection of both curves opens a regime of universal behavior, independent of the disorder model, $L$, and $l$. As expected, localized states become unstable at high density and disorder where the value $p \approx 2$ indicates a collapse of nearly all states to two-body resonances (dark purple region).

\section{DISCUSSION}

In the past few years, a new experimental light-matter platform has emerged, where a single mode of a subwavelength waveguide (nanoguide) is coupled to various materials, ranging from gas-phase atoms [19-21], semiconductor quantum dots [16-18,49], molecules [15], and even superconducting qubits $[24,25]$. The efficient coupling of the individual quantum emitters to the optical mode of the nanoguide and the ability to register the positions and transition frequencies of the individual emitters with very high spatial and spectral resolution make nanoguides ideally suited for investigating fundamental features of light propagation in dielectric media. On the theoretical side, these systems have been studied in the context of Bragg cavities [42], coupling of two atoms via a photonic channel [40] or realization of photon-photon interactions via an atom $[42,50]$. These features promote nanoguides as a very promising avenue for exploring polaritonic physics [7], light localization [31], and related phenomena [51] in a compact and scalable fashion.

Our analysis shows that nanoguide-mediated multiple scattering among emitters can give rise to normal-mode splitting even without a cavity or a plasmonic structure. The key parameters for this phenomenon are the spatial mode coupling efficiency $\beta$ and the spectral bandwidth, which determine the coupling between the emitters and a photon. As in the case of the microcavity-based mode splittings [2], however, shortcomings in these quantities can be compensated by increasing the number of emitters within a photon coherence length.

We remark that the effects reported in this work cannot be achieved in an open three-dimensional (bulk) ensemble because of the poor mode matching between the scattering and the incident field. We have also shown that each realization of an ensemble of emitters may contain many spectral features that arise from near-field dipole-dipole interactions or from state localization caused by disorder. Interestingly, these persist even in the presence of photon loss from the nanoguide. Moreover, the most super-radiant states, which determine the optical response of the nanoguide and the spectral mode splitting, are robust to moderate disorder. A phase diagram summarizes different regimes and captures the impact of density and disorder on the polaritonic state properties.
By not invoking a quantum optical treatment, we have aimed to emphasize the central role of the spatial mode matching for achieving collective many-body states. However, our semiclassical approach captures well the essence of a quantum-optical master equation at weak driving fields and can be readily extended to multilevel emitters [52] by using a modified polarizability. Furthermore, the model can be extended to account for more complex waveguide polarization modes, angular momentum states, and their impact on the propagation [53]. Moreover, it is possible to include different models of disorder [29] with contributions of clusters beyond pairs and excluded volumes [54]. Similarly, one might also account for inhomogeneous spectral distributions, as commonly encountered in solid-state systems, or advanced models of dephasing.

\section{ACKNOWLEDGMENTS}

We thank D. Martín-Cano, I. Chremmos, and P. Türschmann for helpful comments. V.S. also acknowledges fruitful discussions with J.-J. Greffet. This work was supported by an Alexander von Humboldt professorship, the Max Planck Society and the European Research Council (Advanced Grant SINGLEION).

\section{APPENDIX A: SCATTERERS AND NANOGUIDES}

\section{Scatterers}

For the numerical simulations the system exposed in Sec. II was taken to be a cylindrical nanoguide that contains two-level emitters with transitions at $760 \mathrm{~nm}$ and a natural linewidth of $\Gamma_{0}=400 \mathrm{MHz}$, inspired by well-aligned molecular emitters in solid-state environments $[15,55]$. Imposing modified atomic units, the transition dipole matrix element is $|\mu|=\hbar=1$, and the polarizability in the near-resonant approximation is $\alpha(\Delta)=\frac{1}{\Delta-i \Gamma / 2}$, with $\Gamma=\Gamma_{0}+\Gamma_{\text {deph }}$. Dephasing at a rate $\Gamma_{\text {deph }}$ is only included when explicitly mentioned.

\section{Green's function}

The dielectric cylindrical nanoguide was taken to have radius $R=160, \ldots, 215 \mathrm{~nm}$ and refractive index $n=1.5$ surrounded by vacuum. Modeling of the photon propagation requires the solution of the full 3D-electromagnetic boundaryvalue problem. However, near the cylinder axis, only radial dipoles couple efficiently to guided photons, motivating the projection of all dipoles, polarizabilities, fields, etc. on the radial axis, resulting in scalar equations. The radially projected dyadic Green's function in the cylinder describes the radial field generated by a radial dipole $d_{n}$ placed on the axis in $z_{n}$,

$$
E_{n}^{\operatorname{dip}}(z)=G\left(z-z_{n}\right) d_{n} .
$$

It is expressed in the scattering decomposition $G(z)=$ $G^{(\mathrm{free})}(z)+G^{(\mathrm{sc})}(z)$, where the free contribution

$$
G^{(\mathrm{free})}(z)=-\frac{e^{i \kappa z}}{4 \pi \kappa^{2} z^{3}}\left(1-i \kappa z+\kappa^{2} z^{2}\right)
$$

describes the field distribution in free space that dominates in the near field. Scattering off the cylinder walls provides a contribution that includes the impact of guided modes and 
dominates at large distances. For radially aligned emitters on the axis the well-known decomposition $[33,41]$ simplifies to

$$
G^{(\mathrm{sc})}(z)=i \int \frac{d h}{16 \pi} e^{i h z}\left[r_{M M}-\frac{2 i h}{k_{2}} r_{M N}+\frac{h^{2}}{k_{2}^{2}} r_{N N}\right] \text {. }
$$

Here, $\kappa_{1}=\omega / c$ and $\kappa_{2}=n \omega / c$ denote the propagation constant in vacuum or the fiber medium, respectively, and we introduced reflection coefficients

$$
r_{\alpha}=-\frac{H_{1}^{(1)}\left(x_{2}\right)}{J_{1}\left(x_{2}\right)} \frac{A+B_{\alpha}}{A+C}
$$

with

$$
\begin{aligned}
A & =-(R \omega / c)^{2}(h R)^{2}\left(1-n^{2}\right)^{2} /\left(x_{1}^{2} x_{2}^{2}\right), \\
C & =\left[\tilde{h}\left(x_{1}\right) x_{2}-\tilde{j}\left(x_{2}\right) x_{1}\right]\left[\tilde{h}\left(x_{1}\right) x_{2}-n^{2} \tilde{j}\left(x_{2}\right) x_{1}\right], \\
B_{M M} & =\left[\tilde{h}\left(x_{1}\right) x_{2}-\tilde{h}\left(x_{2}\right) x_{1}\right]\left[\tilde{h}\left(x_{1}\right) x_{2}-n^{2} \tilde{j}\left(x_{2}\right) x_{1}\right], \\
B_{N N} & =\left[\tilde{h}\left(x_{1}\right) x_{2}-\tilde{j}\left(x_{2}\right) x_{1}\right]\left[\tilde{h}\left(x_{1}\right) x_{2}-n^{2} \tilde{h}\left(x_{2}\right) x_{1}\right], \\
B_{M N} & =-A+i\left(R^{2} h \kappa_{2}\right)\left(n^{2}-1\right)\left[\tilde{h}\left(x_{2}\right)-\tilde{j}\left(x_{2}\right)\right] .
\end{aligned}
$$

Here, $x_{i}=R \sqrt{\kappa_{i}^{2}-h^{2}}, \tilde{h}(x)=\frac{d}{d x} \ln H_{1}^{(1)}(x)$, and $\tilde{j}(x)=$ $\frac{d}{d x} \ln J^{(1)}(x) . J^{(1)}(x)$ and $H_{1}^{(1)}(x)$ denote the Bessel function and the Hankel function of the first kind. The integral is evaluated numerically.

\section{Single-mode regime}

In the single-mode regime, the guided mode contribution with wave number $k=n_{\mathrm{eff}} \omega / c$ can be extracted from the resonances of the scattered term $G^{(\mathrm{sc})}$ and yield the asymptotic far-field $[40,41]$

$$
G(z) \approx i \beta \operatorname{Im}[G(0)] \exp (i k|z|) .
$$

The amplitude was expressed in terms of the local-field corrected single-emitter linewidth $\Gamma_{0}=2 \operatorname{Im}[G(0)]$, and includes the radiation reaction [32] in the nanoguide environment. For the above parameters, the coupling efficiency of a single emitter to the guided mode is $\beta=\lim _{z \rightarrow \infty}|G(z)| / \operatorname{Im}[G(0)] \approx$ $0.4, \ldots, 0.56$. Different choices of the radius or material, e.g., IR-emitters inside a semiconductor cylinder (GaAs, $R=140 \mathrm{~nm}$, transition at $1.2 \mu \mathrm{m}$ ) may reach $\beta>0.9$ [56]. Note that over the relevant narrow frequency band of a few hundred $\Gamma_{0}$, waveguide dispersion is negligible, as can be seen by the vertical black curve in Figs. 2(e) and 2(f).

\section{APPENDIX B: MEAN-FIELD APPROXIMATION}

In long dense chains $(k L \ll 1 \ll N k L)$, a mean-field treatment following the approach of Ref. [57] can give a good understanding of the physics, especially when dephasing limits the impact of coherent multiple scattering. We rewrite Eq. (2) of the main text for the fields, assuming a homogeneous dipole line density $\rho(z)=N / Z$ over the system length $Z=N L$

$$
E(z)=E_{0}(z)+\int_{0}^{Z} d z^{\prime} \alpha \rho\left(z^{\prime}\right) G\left(z-z^{\prime}\right) E\left(z^{\prime}\right) .
$$

Since most of the emitters are coupled via the far field, we neglect near fields and use Eq. (A6). The good agreement with the numerical results justifies a posteriori the assumption that nearby emitters mainly induce a global frequency shift. Replacing the total field by a mean-field $E^{\text {tot }}(z)=E_{+} e^{i q z}+$ $E_{-} e^{-i q z}$, the integrals can be performed, considering the discontinuity of $G$ at $z=z^{\prime}$

$$
\begin{aligned}
E_{+} e^{i q z}+E_{-} e^{-i q z}= & E_{0} e^{i k z}+\frac{N \beta}{Z} \alpha \operatorname{Im}[G(0)]\left[-e^{i q z}\left(\frac{E_{+}}{k-q}+\frac{E_{+}}{q+k}\right)-e^{-i q z}\left(\frac{E_{-}}{k-q}+\frac{E_{-}}{k+q}\right)\right. \\
& \left.+e^{-i k z}\left(\frac{E_{+} e^{i(k+q) Z}}{k+q}+\frac{E_{-} e^{i(k-q) Z}}{k-q}\right)+e^{i k z}\left(\frac{E_{-}}{k+q}+\frac{E_{+}}{k-q}\right)\right] .
\end{aligned}
$$

For self-consistency, the coefficients for each exponential must balance. Importantly, for $e^{ \pm i q z}$ we have

$$
1+\frac{N \beta}{Z} \alpha \operatorname{Im}[G(0)]\left(\frac{1}{k-q}+\frac{1}{k+q}\right)=0,
$$

from which we obtain the effective wave number in the filled waveguide

$$
q^{2}=k^{2}+2 k \frac{N \beta}{Z} \alpha \operatorname{Im}[G(0)] .
$$

For the case of Fig. 2(e), the resulting dispersion relation is given by the white dashed curve, taking into account the background shift. Finally, we define a dressed effective mode index $\tilde{n}=q c / \omega=n_{\text {eff }} q / k$ that fulfills a generalized Clausius-Mossotti relation [57] when expanded at lowest order in $\alpha$

$$
\frac{\tilde{n}-1}{\tilde{n}+2} \approx \frac{2 \alpha}{3 k} \frac{N}{Z} \beta \operatorname{Im}[G(0)]=\frac{\tilde{\alpha}}{3 \varepsilon_{0}} \frac{1}{L A_{\mathrm{eff}} n_{\mathrm{eff}}} .
$$

To obtain the second form, we use

$$
\beta \operatorname{Im}[G(0)]=\Gamma_{0} / \Gamma_{\text {bulk }} \operatorname{Im}\left[G_{\text {bulk }}(0)\right] \approx \frac{\omega / c}{2 \varepsilon_{0} A_{\text {eff }}}
$$

with the effective area $A_{\text {eff }}=\frac{v_{g} n^{2}}{c} \frac{\int d^{2} \boldsymbol{r} \varepsilon(\boldsymbol{r})\left|\boldsymbol{E}_{\text {mode }}(\boldsymbol{r})\right|^{2}}{\varepsilon(\mathbf{0})\left|\boldsymbol{E}_{\text {mode }}(\mathbf{0})\right|^{2}}$, the transverse field distribution of the mode $E_{\text {mode }}(\boldsymbol{r})$, and its group velocity $v_{g}$ [58]. This underlines that the density needs to be averaged over the mode volume rather than the geometric cylinder. Note that at this order, terms related to a local exclusion volume [57] can be neglected.

\section{APPENDIX C: TWO-BODY RESONANCES}

The dark and bright resonances of two-coupled emitters are obtained by direct diagonalization of the matrix $\mathcal{A}^{-1}$ in Eq. (3) for $N=2$, yielding

$$
\delta_{ \pm}^{(2)}-i \gamma_{ \pm}^{(2)} / 2=\Delta-[i \Gamma / 2 \pm G(\Delta z, \omega)] .
$$


Varying the emitter distance $0<\Delta z<\infty$, these eigenvalues cover the green curve in the complex plane in Fig. 3(f). At large separations, when the interaction is mediated by the waveguide mode alone, Eq. (A6) limits the amplitude $|G|=\beta \Gamma_{0} / 2$ and thus bounds both the mode splitting and the line widths.

In the case of near-field coupling, the waveguide mode $E^{\operatorname{ext}}\left(z_{n}\right) \approx E_{0}$ can only drive the symmetric eigenstate $\Psi_{+}$, inducing dipoles

$$
d_{n}=\sum_{m=1}^{2} \mathcal{A}_{n m} E_{m}=\frac{2 E_{0}}{\Delta-i \Gamma / 2-G(\Delta z)} .
$$

We find the pair-transmission amplitude

$$
T_{2}=\left|1+\frac{2 i \beta \Gamma / 2}{\Delta-i \Gamma / 2-G(\Delta z)}\right|^{2}=|1-r|^{2} .
$$

The eigenvectors are $\Psi_{ \pm}^{(2)}=( \pm 1,1) / \sqrt{2}$. On the bright resonance $(\Delta-\operatorname{Re}[G(\Delta z)] \approx 0)$ and in near-field coupling regime $(\operatorname{Im}[G(\Delta z)] \approx \Gamma / 2), T_{2}=(1-\beta)^{2}$ becomes identical to the one of a single resonant emitter. Besides, the form of the two-body potential induces a correlation between line shift and linewidth: bright states are blue-shifted $(-\Delta=\delta>0)$ with respect to the single resonance, and only these can provide significant extinction. From Eq. (C3) it is clear that the scattering is reduced and $r \rightarrow 0$ for red-shifted detuning $(-\Delta<0)$.

In large ensembles, the states of a chain of $N=2$ can nonetheless provide an excellent understanding of the underlying physics: this is the case, when in the regime of disordered near-field coupled chains, where the interaction to one closest neighbor dominates for each emitter. In this case, $\mathcal{A}^{-1}$ approximately factorizes into a product of $2 \times 2$ matrices, which may be diagonalized individually so that each pair submatrix gives rise to one pair of dark and bright states. Similar states have been encountered in 3D ensembles of many emitters $[46,47]$ but have more drastic impacts on linear arrangements.

\section{APPENDIX D: BRAGG RESONANCES AND LINEWIDTH LIMITS}

We outline the calculation of the collective modes on a Bragg resonance $k L / \pi \in \mathbb{N}$ and assume even $N$. The latter restriction has, however, little impact at the large $N$ and finite $\beta<1$ considered here. In the far-field approximation,

$$
\mathcal{A}_{m n}^{-1} \approx \frac{\delta_{m n}}{\alpha_{n}}-i \beta \frac{\Gamma}{2} \operatorname{Im}[G(0)] e^{i k L|m-n|}\left(1-\delta_{m n}\right),
$$

which is a circulant matrix [59] and can be diagonalized by Fourier transformation. The eigenvalues are

$$
\delta_{m}-i \frac{\gamma_{m}}{2} \approx \frac{1}{\alpha}-i \beta \frac{\Gamma}{2} \frac{e^{-i(2 \pi m / N-k L)(N-1)}-1}{1-e^{i(\pi m / N-k L)}} .
$$

For even (odd) values of $k L / \pi$ a pole is encountered at $m=0(m=N / 2)$. L'Hôpital's rule then provides a single super-radiant (bright) state, while the other $N-1$ states are degenerate and subradiant (dark):

$$
\delta_{m}-i \frac{\gamma_{m}}{2}=\frac{1}{\alpha}-i \beta \frac{\Gamma}{2} \times\left\{\begin{array}{ll}
-1 & \text { dark } \\
N-1 & \text { bright }
\end{array} .\right.
$$

This agrees with a recent discussion that assumed $\beta=1$ [60]. The mode functions are $(1 \leqslant j \leqslant N)$

$$
\boldsymbol{\psi}_{m, j} \approx e^{-2 \pi i m(j-1) / N} / \sqrt{N}
$$

Only the single bright mode $\Psi_{+}=(1, \pm 1,1, \pm 1, \ldots) / \sqrt{N}$ matches the periodicity of the guided mode $\left[E^{\operatorname{ext}}\left(z_{n}\right)=\right.$ $\left.E_{0} e^{i k z_{n}}=E_{0} \Psi_{+, n}\right]$, whereas the other modes have zero mode overlap. To find the scattering matrix element, we neglect the dark modes by projecting onto $\Psi_{+}$, so that

$$
\mathcal{A}^{-1} \approx \Delta-i \frac{\Gamma}{2}[1+\beta(N-1)] \Psi_{+} \otimes \Psi_{+} .
$$

In the collective basis, the inversion is obvious. Far from the source region Eq. (A6) applies and

$$
\begin{aligned}
E^{\mathrm{tot}}(z) & =E^{\mathrm{ext}}(z)+\sum_{n, m} G\left(z-z_{n}\right) \mathcal{A}_{n m} E^{\mathrm{ext}}\left(z_{m}\right) \\
& =E_{0} e^{i k z}+\sum_{n} \frac{i \beta \frac{\Gamma}{2} e^{i k\left|z-z_{n}\right|}}{\Delta-i[1+\beta(N-1)] \Gamma / 2} .
\end{aligned}
$$

In the forward direction $\left(z \gg z_{N}>0, z_{n}=2 \pi k L\right)$, we recover the transmitted power fraction [42]

$$
\mathcal{T}=\frac{\left|E^{\mathrm{tot}}\right|^{2}}{\left|E_{0}\right|^{2}}=\left|1+\frac{i N \beta \Gamma / 2}{\Delta-i[1+\beta(N-1)] \Gamma / 2}\right|^{2} .
$$

At the single-body resonance $\Delta=0$ we find $\mathcal{T}=|1-r|^{2}$, $\mathcal{R}=|r|^{2}, \mathcal{L}=1-\mathcal{R}-\mathcal{T}, r=N \beta /[N \beta+(1-\beta)]$. Equation (D6) can be solved numerically away from the Bragg resonance condition.

Section IV shows that the values $\gamma_{ \pm}$from Eq. (D3) apply, as long as Eq. (D1) holds. For $\gamma_{+}$, this follows also from the matrix norm (operator norm) $\left\|\mathcal{A}^{-1}\right\|=\max \{\mid \epsilon-$ $i \gamma / 2 \mid\}) \equiv \max _{n}\left\{\sum_{m=1}^{N}\left|\mathcal{A}_{n m}^{-1}\right|\right\}$. The off-diagonal entries have an amplitude $\beta$, so that the norm suggests an extreme eigenvalue $\left\|\mathcal{A}^{-1}(\Delta=0)\right\|=[(N-1) \beta+1] \Gamma / 2=\gamma_{+} / 2$, exactly realized by the bright state at a Bragg resonance and confirming its universal character.
[1] J.-M. Raimond and S. Haroche, Exploring the Quantum (Oxford University Press, Oxford, 2006).

[2] P. Törmä and W. L. Barnes, Rep. Prog. Phys. 78, 013901 (2015).

[3] C. Weisbuch, M. Nishioka, A. Ishikawa, and Y. Arakawa, Phys. Rev. Lett. 69, 3314 (1992).
[4] D. Lidzey, D. D. C. Bradley, M. S. Skolnick, T. Virgili, S. Walker, and D. M. Whittaker, Nat. (London) 395, 53 (1998).

[5] S. Kéna-Cohen, M. Davanco, and S. R. Forrest, Phys. Rev. Lett. 101, 116401 (2008).

[6] T. Schwartz, J. A. Hutchison, J. Leonard, C. Genet, S. Haacke, and T. W. Ebbesen, ChemPhysChem 14, 125 (2012). 
[7] J. Keeling, F. M. Marchetti, M. H. Szymanska, and P. B. Littlewood, Semicond. Sci. Technol. 22, R1 (2007).

[8] T. K. Hakala, J. J. Toppari, A. Kuzyk, M. Pettersson, H. Tikkanen, H. Kunttu, and P. Törmä, Phys. Rev. Lett. 103, 053602 (2009).

[9] G. Wrigge, I. Gerhardt, J. Hwang, G. Zumofen, and V. Sandoghdar, Nat. Phys. 4, 60 (2008).

[10] G. Zumofen, N. M. Mojarad, V. Sandoghdar, and M. Agio, Phys. Rev. Lett. 101, 180404 (2008).

[11] K. G. Lee, X. W. Chen, H. Eghlidi, P. Kukura, R. Lettow, A. Renn, V. Sandoghdar, and S. Götzinger, Nat. Photon. 5, 166 (2011).

[12] X.-L. Chu, T. J. K. Brenner, X.-W. Chen, Y. Ghosh, J. A. Hollingsworth, V. Sandoghdar, and S. Götzinger, Optica 1, 203 (2014).

[13] V. I. Balykin, K. Hakuta, F. Le Kien, J. Q. Liang, and M. Morinaga, Phys. Rev. A 70, 011401(R) (2004).

[14] J. T. Shen and S. Fan, Opt. Lett. 30, 2001 (2005).

[15] S. Faez, P. Türschmann, H. R. Haakh, S. Götzinger, and V. Sandoghdar, Phys. Rev. Lett. 113, 213601 (2014).

[16] A. V. Akimov, A. Mukherjee, C. L. Yu, D. E. Chang, A. S. Zibrov, P. R. Hemmer, H. Park, and M. D. Lukin, Nat. (London) 450, 402 (2007).

[17] T. Lund-Hansen, S. Stobbe, B. Julsgaard, H. Thyrrestrup, T. Sünner, M. Kamp, A. Forchel, and P. Lodahl, Phys. Rev. Lett. 101, 113903 (2008).

[18] R. Yalla, F. Le Kien, M. Morinaga, and K. Hakuta, Phys. Rev. Lett. 109, 063602 (2012).

[19] E. Vetsch, D. Reitz, G. Sagué, R. Schmidt, S. T. Dawkins, and A. Rauschenbeutel, Phys. Rev. Lett. 104, 203603 (2010).

[20] A. Goban, C.-L. Hung, S.-P. Yu, J. Hood, J. Muniz, J. Lee, M. Martin, A. McClung, K. Choi, D. Chang, O. Painter, and H. J. Kimble, Nat. Comm. 5, 3808 (2014).

[21] N. V. Corzo, B. Gouraud, A. Chandra, A. Goban, A. S. Sheremet, D. V. Kupriyanov, and J. Laurat, Phys. Rev. Lett. 117, 133603 (2016).

[22] R. Kolesov, B. Grotz, G. Balasubramanian, R. J. Stöhr, A. A. L. Nicolet, P. R. Hemmer, F. Jelezko, and J. Wrachtrup, Nat. Phys. 5, 470 (2009).

[23] A. Stiebeiner, O. Rehband, R. Garcia-Fernandez, and A. Rauschenbeutel, Opt. Express 17, 21704 (2009).

[24] O. Astafiev, A. M. Zagoskin, A. A. Abdumalikov, Y. A. Pashkin, T. Yamamoto, K. Inomata, Y. Nakamura, and J. S. Tsai, Science 327, 840 (2010).

[25] A. F. van Loo, A. Fedorov, K. Lalumière, B. C. Sanders, A. Blais, and A. Wallraff, Science 342, 1494 (2013).

[26] L. L. Foldy, Phys. Rev. 67, 107 (1945).

[27] M. Lax, Rev. Mod. Phys. 23, 287 (1951).

[28] A. Lagendijk and B. van Tiggelen, Phys. Rep. 270, 143 (1996).

[29] B. Kramer and A. MacKinnon, Rep. Prog. Phys. 56, 1469 (1993).

[30] S. John, Phys. Today 44, 32 (1991).

[31] A. Lagendijk, B. van Tiggelen, and D. S. Wiersma, Phys. Today 62, 24 (2009).

[32] P. de Vries, D. van Coevorden, and A. Lagendijk, Rev. Mod. Phys. 70, 447 (1998).
[33] L. W. Li, M. S. Leong, T. S. Yeo, and P. S. Kooi, J. Electromagn. Waves. Appl. 14, 961 (2000).

[34] E. Rephaeli, Ş. E. Kocabaş, and S. Fan, Phys. Rev. A 84, 063832 (2011).

[35] D. Martín-Cano, L. Martín-Moreno, F. García-Vidal, and E. Moreno, Nano Lett. 10, 3129 (2010).

[36] A. González-Tudela, P. A. Huidobro, L. Martín-Moreno, C. Tejedor, and F. J. García-Vidal, Phys. Rev. Lett. 110, 126801 (2013).

[37] A. Delga, J. Feist, J. Bravo-Abad, and F. J. Garcia-Vidal, Phys. Rev. Lett. 112, 253601 (2014).

[38] Spontaneous emission and laser oscillation in microcavities, edited by H. Yokoyama and K. Ujihara (CRC Press, Boca Raton, 1995).

[39] X. Deng, B. L. Altshuler, G. V. Shlyapnikov, and L. Santos, Phys. Rev. Lett. 117, 020401 (2016).

[40] A. González-Tudela, D. Martin-Cano, E. Moreno, L. MartinMoreno, C. Tejedor, and F. J. Garcia-Vidal, Phys. Rev. Lett. 106, 020501 (2011).

[41] H. R. Haakh and S. Scheel, Phys. Rev. A 91, 052707 (2015).

[42] D. E. Chang, L. Jiang, A. V. Gorshkov, and H. J. Kimble, New J. Phys. 14, 063003 (2012).

[43] K. Y. Bliokh, Y. P. Bliokh, V. Freilikher, A. Z. Genack, B. Hu, and P. Sebbah, Phys. Rev. Lett. 97, 243904 (2006).

[44] E. Abrahams, P. W. Anderson, D. C. Licciardello, and T. V. Ramakrishnan, Phys. Rev. Lett. 42, 673 (1979).

[45] T. Jonckheere, C. A. Müller, R. Kaiser, C. Miniatura, and D. Delande, Phys. Rev. Lett. 85, 4269 (2000).

[46] S. E. Skipetrov and I. M. Sokolov, Phys. Rev. Lett. 112, 023905 (2014).

[47] M. Rusek, J. Mostowski, and A. Orłowski, Phys. Rev. A 61, 022704 (2000).

[48] A. Cazé, R. Pierrat, and R. Carminati, Phys. Rev. A 82, 043823 (2010).

[49] R. Yalla, M. Sadgrove, K. P. Nayak, and K. Hakuta, Phys. Rev. Lett. 113, 143601 (2014).

[50] J. T. Shen and S. Fan, Phys. Rev. Lett. 98, 153003 (2007).

[51] I. Carusotto and C. Ciuti, Rev. Mod. Phys. 85, 299 (2013).

[52] D. Witthaut and A. S. Sørensen, New J. Phys. 12, 043052 (2010).

[53] T. Ramos, H. Pichler, A. J. Daley, and P. Zoller, Phys. Rev. Lett. 113, 237203 (2014).

[54] D. E. Chang, J. I. Cirac, and H. J. Kimble, Phys. Rev. Lett. 110, 113606 (2013).

[55] F. Jelezko, P. Tamarat, B. Lounis, and M. Orrit, J. Phys. Chem. 100, 13892 (1996).

[56] J. Claudon, J. Bleuse, N. S. Malik, M. Bazin, P. Jaffrennou, N. Gregersen, C. Sauvan, P. Lalanne, and J.-M. Gérard, Nat. Photon. 4, 174 (2010).

[57] A. Parola, R. Piazza, and V. Degiorgio, J. Chem. Phys. 141, 124902 (2014).

[58] Y. C. Jun, R. M. Briggs, H. A. Atwater, and M. L. Brongersma, Opt. Express 17, 7479 (2009).

[59] R. M. Gray, Found. Trends Commun. Inf. Theory 2, 155 (2005).

[60] Z. Liao, X. Zeng, S.-Y. Zhu, and M. S. Zubairy, Phys. Rev. A 92, 023806 (2015). 